Como citar este artículo:

Vertiz, J. J., Aparicio, M. F., Guevara, M. F., Díaz, J.Y., Pérez, S. y Menacho, I. (2020). Participación ciudadana como estrategia de diagnóstico para la toma de decisiones para reducir la desnutrición crónica en un distrito de Lima. Revista Eleuthera, 22(2), 132-146. DOI: 10.17151/eleu.2020.22.2.9.

\title{
Participación ciudadana como estrategia de diagnóstico para la toma de decisiones para reducir la desnutrición crónica en un distrito de Lima*
}

\section{Citizen participation as a diagnostic strategy for decision-making to reduce chronic malnutrition in a district of Lima}

\author{
JaCinTO JOAQuín Vertiz-OSOREs** \\ María Fanny Aparicio-Fernández*** \\ Manuel Felipe-Guevara-Duarez**** \\ JUANA YRIS DÍAZ-MUJICA***** \\ Segundo Pérez-SAAVEDRA****** \\ ISABEL MenACHO-VARGAS $* * * * * *$
}

\section{Resumen}

Objetivo. Conocer la pertinencia de la participación ciudadana como estrategia en la toma de decisiones en la reducción de la desnutrición crónica. Metodología. Estudio fenomenológico. Se emplearon entrevistas y focus group. La población consideró ciudadanos padres de familia o cuidadores de niños con desnutrición crónica. La muestra fue de 500 participantes, incluyendo a 40 funcionarios públicos, 25 agentes comunitarios de salud y 18 voluntarios. Resultados. El diálogo ciudadano de salud

\footnotetext{
${ }^{*}$ El estudio fue producto de una investigación fenomenológica realizada en dos Comunidades del Distrito de San Juan de Lurigancho de Lima Metropolitana durante los meses de enero a junio del 2018. Los resultados fueron analizados bajo los criterios del Ministerio de Salud (Minsa), mediante la Dirección de Redes Integradas de Salud, Lima, cuyo propósito es comprender la percepción social en el control y reducción de la desnutrición crónica y la anemia de los niños menores de 5 años. El diálogo ciudadano en salud, como mecanismo de participación ciudadana, responde a una necesidad de la colectividad, pues se lo vincula a partir del proceso de descentralización política que empieza por reconocer los derechos ciudadanos y la asunción de la responsabilidad que le compete al Estado. Con ese considerando, se pretendió medir el nivel de involucramiento de esta población en temáticas conexas a la efectivización de una de las políticas públicas en salud, conllevando a un ejercicio de potenciamiento de las capacidades de los ciudadanos como gestores de su propio sistema salubrista en estratos con severas carencias socioeconómicas y con un gran clamor por el respeto del derecho fundamental de una vida humana digna.

${ }^{* *}$ Universidad Nacional Tecnológica de Lima Sur. Lima, Perú. jvertiz@untels.edu.pe

(D) orcid.org/0000-0003-2774-1207 Google Scholar

**** Escuela de Oficiales de la Fuerza Aérea del Perú. Lima, Perú. maparicio@eofap.edu.pe

(D) orcid.org/0000-0001-7439-3036 Google Scholar

***** Universidad Nacional Amazónica de Madre de Dios. Puerto Maldonado, Perú. E-mail: mguevara@unamad.edu.pe (D) orcid.org/0000-0001-7266-0508 Google Scholar

******* Universidad Privada de Huancayo Franklin Roosvelt. Junin, Perú. E-mail: ydiaz@uroosevelt.edu.pe

(D) orcid.org/0000-0001-8268-4626 Google Scholar

******* Universidad César Vallejo. Lima, Perú. sperezs@ucv.edu.pe.

(D) orcid.org/0000-0002-2366-6724 Google Scholar

******** Universidad César Vallejo. Lima, Perú. E-mail: isabelmenachov@gmail.com.

(D) orcid.org/0000-0001-6246-4618 Google Scholar
} 
permitió recopilar la opinión de la población, consolidándose como una metodología de gestión. Se tuvo un diagnóstico sobre los determinantes de la alimentación, los mismos que se relacionan con los mitos colectivos, comunicación e información, pobreza y factores culturales. Conclusión. Es pertinente la participación ciudadana como estrategia en la toma de decisiones por parte del Estado, directivos o funcionarios públicos, porque es un medio efectivo de comunicación entre ambos.

Palabras clave: participación ciudadana, desnutrición, toma de decisiones.

\section{Abstract}

Objective: To recognize the relevance of citizen participation as a strategy in decision-making for reducing chronic malnutrition. Methodology: Phenomenological study. Interviews and focus group were used. The population included citizens who were parents and/or caregivers of children with chronic malnutrition. The sample involved 500 participants, including 40 public officials, 25 community health agents and 18 volunteers. Results: The citizen health dialogue allowed gathering the opinion of the population, consolidating itself as a management methodology. A diagnosis on the determinants of food which are related to collective myths, communication and information, poverty and cultural factors was made. Conclusion: Citizen participation is relevant as a strategy in decision-making by the State, managers or public officials, because it is an effective means of communication between both groups.

Key words: citizen participation, malnutrition, decision making.

\section{Introducción}

El Estado es un consenso social (Halperin, 2003), en él se establecen múltiples dinámicas: económicas, sociales, estructurales y políticas, puesto que se participa en democracia (Sartori, 2009). En la organización estatal, la participación ciudadana es fundamental; se rige bajo diversas formas participativas en las decisiones y gestión estatal. A partir del proceso de la descentralización, en la región latinoamericana se incorporan estructuralmente los mecanismos de participación ciudadana en la gestión pública (Ward, Cafiero, Fretigny, Cogrove \& Seror, 2019), extendiéndose a países de otras latitudes (Serapioni \& Matos, 2014; Padmaja, Pramanik, Pingali, Bantilan \& Kavitha, 2019). Así, en las últimas décadas, Perú incorporó mecanismos de participación ciudadana como parte de la gestión pública (Ooms, Keygnaert \& Hammonds, 2019) configurándose en el régimen constitucional a los presupuestos municipales (Ley Marco del Presupuesto Participativo, Congreso de la República, 2003) y en los planes de desarrollo concertados, entre otras dinámicas socioeconómicas vigentes (Sistema Peruano de Información Jurídica, 2003).

La participación ciudadana como estrategia en la toma de decisiones para la reducción de la desnutrición crónica (Ngo \& Serra-Majem, 2017, Mearin, 2018), responde a necesidades de 
Participación ciudadana como estrategia de diagnóstico para la toma de decisiones para reducir la desnutrición crónica en un distrito de Lima

la población, derivadas del reconocimiento de sus derechos ciudadanos y la responsabilidad del Estado al involucrar a la población en temas relacionados a las políticas públicas de salud (Sabbahi, Li, Davis \& Downs, 2018). Esto conlleva a un ejercicio de mejoramiento de capacidades en los derechos ciudadanos de la salud, tanto para los representantes de la sociedad civil como para los operadores de salud y autoridades de todo nivel de gobierno con el objeto de que se constituyan en espacios propositivos y de inclusión social, respecto a los derechos de las personas usuarias de estos servicios elementales (Congreso de la República, 2003). A pesar de que algunos estudios manifiestan que las buenas prácticas de participación ciudadana están relacionadas a las creencias y actitudes de la ciudadanía, profesionales, sistemas sanitarios y contexto social (Delamaza, 2011; Gómez, Pastor, Pérez e Iriarte, 2017; Franco, Diez, Gullón, Margolles, Cofiño, Pasarín, \& Borrell, 2018).

Para la reducción de la desnutrición crónica infantil se ha considerado una política nacional como estrategia de solución, debido a la indicación expresa que tiene la Organización Mundial de la Salud (WHO, 2014) al declararla como problema de salud pública, teniendo como principales afectados a poblaciones vulnerables: ancianos, mujeres embarazadas y principalmente niños/as menores de cinco años de edad. En ese marco, se generan espacios de diálogo social que buscan el desarrollo del territorio y se concatenan a la mejora de la calidad de vida de la población (Hintze, 2017), desplegando esfuerzos gubernamentales desde hace un poco más de 20 años; y, aunque hay mejoras, los indicadores de logro de metas aún no revelan cumplimiento de las expectativas nacionales, a pesar de la incursión de los gobiernos locales como actores directos de la ejecución de esta política en la actualidad (López, Segura, Pires, Malmusi, Vergara \& Perez, 2018).

De esa manera, el uso de los micronutrientes en familias con niños/as menores de los cinco años es una estrategia medular (Aparco, Bautista, Astete y Pillaca, 2016; Reeves \& Mackenbach, 2019) para fortalecer el Programa de Articulado Nutricional (PAN) e implementar políticas públicas con el involucramiento del gobierno local y diversos actores decisores políticos (López et al., 2018), teniendo resultados que proveen variaciones alentadoras debido al efecto fisiológico casi inmediato del consumo de algunos de estos complementos nutricionales (Cediel Olivares, Brito, Cori \& López, 2015), aunque la resistencia de las familias hacia los cambios de hábitos nutricionales y estilos de vida no proporcionan ninguna garantía de la sostenibilidad del programa, lo que conlleva a pensar en la necesidad de adoptar medidas que intervengan con la toma de decisiones de la población (Martínez y Fernández, 2009).

En efecto, la toma de decisiones es una capacidad propiamente humana (Simon, 1984), dando espacio al poder y la razón de la voluntad que van en una misma dirección; llevando a cabo un proceso de análisis, organización y planificación. Para las gerencias es fundamental que en la planificación se mantenga coherencia con la toma de decisiones (Rodríguez, Pedraja y Araneda, 2013; Bonome, 2009) y, consecuentemente, su eficiencia lleve al éxito, mediante la 
articulación de estrategias, técnicas, procedimientos, y saberes previos que permitan tomar decisiones dentro de la organización (Castro, 2014). En este sentido, la evaluación nutricional incorpora técnicas tradicionales y nuevas metodologías a una unificada, fundamentada y racional forma de conocer el estado nutricional de los pacientes (Jo, Marín y Puicón, 2018). Para ello, es necesario que los profesionales estén entrenados para laborar en el área de nutrición, pues, como lo afirman Ravasco, Anderson y Mardones (2010), esta tarea no solo es competencia de nutricionistas, sino que es un programa integral que involucra a las demás profesiones, como se precisó en la Declaración de Cancún en el 2008 (Felanpe, 2008; De la Cruz, Figueredo, Dugloszewski, Díaz, Spolidoro \& Mato, 2008).

Desde la teoría social de Thomas (como se citó en Timasheff, 1961), las unidades fundamentales de la realidad social son actitudes y valores que repercuten en la conducta de los individuos cuando están en situación de riesgo. Por tanto, esta situación exige que ante una realidad como la desnutrición crónica (circunstancia objetiva), el individuo se vea en la necesidad de incluir reglas o patrones culturales de abordaje y su actitud cambie (conducta social). En efecto, la actitud es la tendencia a obrar de un modo y no de otro, representando así un deseo de mejorar la situación en la que está. Por otro lado, la desnutrición crónica exige analizarla desde la lógica de la teoría de la racionalidad para la toma de decisiones de Simón (1984), considerando que la correcta decisión, tomada por la gerencia, debería contar con conocimientos, amplia experiencia en el tema, ética y seguridad personal (Koontz \& Weihrich, 2013); así se garantizaría que dicha decisión tenga beneficio, no solamente para la institución, sino también para los sujetos a los cuales tenga cobertura, teniendo como principio rector al bienestar colectivo desde la individualidad (Seligman, 2011). Si bien toda decisión implica un contexto de poder que presupone su cumplimiento, es este mismo poder el que permite el nacimiento de toda política (policy) (Lasswell, 1974). Frente a este contexto teórico, la realidad es un reflejo de las múltiples miradas de los tratadistas, no obstante, la dinámica evolutiva muchas veces no permite predecir algún comportamiento colectivo, por lo que, partiendo de la importancia de explicar la poca eficacia de la política nacional de lucha contra la desnutrición crónica en Perú, se plantea un escenario en donde se consideraría a la participación ciudadana como estrategia de diagnóstico que permitiría disponer de información previa, a emplearse como insumo para una mejor toma de decisiones por los encargados de la ejecución de la política de lucha contra la desnutrición crónica. Este planteamiento busca ser un modelo de trabajo político-social, para lo cual se eligió un contexto socio cultural que tiene uno de los más altos indicadores de desnutrición crónica de Lima Metropolitana, con una alta brecha socioeconómica y una considerable proporción de multiculturalidad poblacional (Matos, 2012). Considerándose como uno de los distritos más grandes del Perú, San Juan de Lurigancho es el escenario donde se ejecutó esta investigación, interviniéndose a personas procedentes de familias que radican allí hace más de 40 años (INEI, 2017). 
Participación ciudadana como estrategia de diagnóstico para la toma de decisiones para reducir la desnutrición crónica en un distrito de Lima

\section{Referente teórico y conceptual}

Para Font, Blanco, Gomá y Jarque (2000), la participación ciudadana consiste en un conjunto de formas y modos mediante la cual una población participan en las decisiones de los actores gubernamentales, debe anotarse que esta participación no necesariamente involucra una participación en la administración pública, sino que puede darse de manera independiente. Por otro lado, también se señala que la participación ciudadana no se reduce a los procesos democráticos de elección de autoridades, sino que involucran acciones de expresión en espacios donde hay libertad de opinión, creatividad y propuestas con responsabilidad y racionalidad (Font et al., 2000, pp. 57-58). Al mirar la participación ciudadana en Perú, en la actualidad, el país cuenta con un marco legal que promueve la participación ciudadana (Congreso de la República, 2002, 2003. Ley $\mathrm{N}^{\circ} 27783$, Ley $\mathrm{N}^{\circ} 27867$, Ley $\mathrm{N}^{\circ} 27972$, Ley $\mathrm{N}^{\circ}$ 26300), en las decisiones y la gestión del Estado, lo cual está expresado en su constitución, y en las normas del proceso de descentralización en curso desde hace más de una década. Comprende a los tres niveles de gobierno como contexto donde se realizan los debates y se llegan a consensos sobre políticas públicas entre la sociedad civil y el Estado (Hernández, Ruano, Hurtig, Goicolea, San Sebastián \& Flores, 2018; Driss, Mellouli \& Trabelsi, 2019; Hoon Chuah et al., 2018).

Por otra parte, la toma de decisiones se define como un proceso que tiene como resultado una determinación respecto a un asunto en concreto (Broeder, Uiters, Have, Wagemakers \& Schuit, 2017), esta determinación involucra aspectos de elección entre distintas alternativas, en la cual, de manera fundamentada, responsable y deliberada se opta por la mejor. Este proceso incluye conocimiento de información previa, capacidad de análisis, evaluación de posibles resultados y asumir las consecuencias de la decisión tomada (Robbins \& Judge, 2013), así, acorde con estos autores, en un entorno organizacional, la mayoría de las decisiones importantes se ejecutan a través del juicio valorativo, más que por modelos prescriptivos definidos, ya que en definitiva, la toma de decisiones permite identificar y solucionar un proceso de acción útil para un problema particular (Stoner, Freeman \& Gilbert, 2003). Finalmente, Gambara y González (2004), afirmaron que es una decisión respecto a una problemática que implica al menos dos alternativas posibles entre las que el sujeto va a optar. Frente a estas opciones, "existe un conflicto por dos motivos: no se evidencia un orden de preferencias claro y porque las consecuencias de los resultados o los cursos de acción a implementar no siempre son óptimamente conocidos" (p. 38).

Por otro lado, la desnutrición crónica es una condición en la cual el nivel de almacenamiento orgánico de nutrientes que el organismo haya acumulado mediante la ingestión de alimentos se agota debido a una deficiencia de calorías, vitaminas y proteínas (Unicef, 2015). Las consecuencias de esta condición fisiológica se visualizan en un retraso del crecimiento corporal a nivel de feto, infante, niño y adolescente (Ravasco et al., 2010). Se debe precisar que la evaluación nutricional de un individuo o de una población es una etapa trascendental en la que 
el personal encargado debe contar con entrenamiento profesional. Esto conlleva a deducir que un correcto empleo de las técnicas y metodologías de evaluación del estado nutricional, demanda no solamente de conocimiento científico y experticia empírica, sino también del desarrollo de actitudes analíticas y sensibilidad humana (Ravasco et al., 2010).

\section{Metodología}

\section{Enfoque metodológico}

Se empleó el enfoque cualitativo, el mismo que opta por la construcción de datos descriptivos para atender un abanico de posibilidades en el camino de la exploración, dando mayor énfasis a la indagación en profundidad para, no solo captar los hechos reales, sino también el avance en la comprensión de los diversos elementos que se relacionan con la problemática de interés (Flick, 2004).

\section{Diseño del estudio}

Se hizo un estudio fenomenológico, caracterizado por centrarse en la experiencia personal. La fenomenología posibilita ir más allá del estudio de los comportamientos observables y controlables, como fenómenos naturales (Heidegger, 2008) vivencias de los sujetos, significados atribuidos, interpretación de procesos entre otras facultades (Flick, 2004). Para ello, se aplicó dos instrumentos: entrevistas (Valles, 1990) y focus group (Barbour, 2013), llevándose a cabo en espacios abiertos de concurrencia pública masiva, espacios que están bajo la dominación de las barriadas (Poloni, 1987).

\section{Escenario de estudio}

El escenario es un sector del distrito más grande y poblado de Lima; San Juan de Lurigancho, cuya población vulnerable alcanza un 25\%, concentrándose principalmente en los sectores de mayor pobreza: Ganímedes, Jaime Zubieta y José Carlos Mariátegui. Allí existen determinantes sociales que generan las causas de la desnutrición crónica infantil (Villar, 2011).

\section{Población y muestra del estudio}

Los actores en la investigación fueron los ciudadanos de a pie, padres de familia o cuidadores de niños con desnutrición crónica. Los participantes fueron protagonistas de la realidad problemática que residen en los tres sectores de mayor pobreza y concentración demográfica. Se seleccionó a 500 participantes, con las condiciones descritas anteriormente, incluyéndose a 40 funcionarios públicos, 25 agentes comunitarios de salud y 18 voluntarios. 
Participación ciudadana como estrategia de diagnóstico para la toma de decisiones para reducir la desnutrición crónica en un distrito de Lima

\section{Aspectos éticos}

El protocolo de estudio se aprobó por el Comité Institucional de Ética e Investigación de la Diris Lima Este. Antes de empezar el estudio, se hicieron las coordinaciones con los diversos actores sociales del distrito: municipalidad distrital, representaste de los parques recreacionales (SERPAR) de Lima, establecimientos de salud, Policía Nacional, otros ministerios, y para la recolección de datos y aplicación de instrumentos se solicitó el consentimiento informado a todos los participantes, explicándose los objetivos y alcances del estudio.

\section{Resultados}

La propuesta del estudio se aproxima a la profundización de tres categorías, focalizadas en los parámetros de una investigación fenomenológica. Los hallazgos encontrados se focalizaron desde la percepción y pertinencia de los participantes: ciudadanos de a pie, preferentemente padres de familia o cuidadores de niños desnutridos menores de cinco años, en el distrito de San Juan de Lurigancho.

Los establecimientos del Ministerio de Salud (Minsa) realizan todos los esfuerzos para bajar la incidencia de la desnutrición crónica, partiendo de la ejecución de las tres unidades de gestión: 'Jaime Zubieta', 'Ganímedes', y 'José Carlos Mariátegui', el incremento de la demanda social supera las expectativas del presupuesto asignado para cubrir las brechas sociales. Por otra parte, el $40 \%$ de la población económicamente activa usa la seguridad social; sin embargo, el seguro social _ EsSalud — no logra satisfacer las necesidades de la población (García y Gálvez, 2016), pese a la distribución de los micronutrientes, muchos de los padres o cuidadores de los niños menores de cinco años no suelen administrarle ese complemento alimenticio en las comidas. Los participantes fueron enfáticos que no solo el Estado debe involucrarse en el problema, sino también los demás actores sociales involucrados directamente con este problema de salud pública en esta localidad.

Los diálogos ciudadanos en salud, si bien es cierto, son oportunos para tomar en cuenta la opinión de la población, debe consolidarse como una metodología de gestión para diseñar no solo los indicadores de desempeño, sino para medir la calidad de las intervenciones estatales frente a ese problema social; permitiendo a su vez otorgar a las madres o cuidadores de niños las herramientas mediante información nutricional y acceso a programas educativos dietéticos no solo dentro de los centros de salud, sino también en otros espacios de mayor concentración pública.

Los determinantes de la alimentación están en relación con los mitos colectivos sobre la nutrición en la dieta de los niños con desnutrición crónica menores de cinco años: "una madre tiene anemia, por tanto, el hijo también"; "no le doy sangrecita, porque soy cristiana evangélica". Por 
otra parte, el consumo de la comida procesada industrialmente no saludable es más aceptada en las familias, porque "la buena comida cuesta caro. La salchipapa es más barata, rápida y sabrosa". En cuanto al acceso de información, los participantes manifestaron sus limitaciones; muchos de ellos no cuentan con las tecnologías de información y comunicación (TIC) a la mano, con excepción de los más jóvenes ya que suelen tener teléfonos móviles, tablets con coberturas a internet; pero se usa mayormente para comunicarse en las redes sociales. En la pobreza, se evidenció que en la mayoría de los casos, ambos padres trabajan, viéndose forzados a encargar a sus hijos a terceros, sean estos familiares, vecinos, centros de cuidados estatales "Wawawasis" o Cunas, o compadres; por lo que "Salen a trabajar y dejan a los niños con alguna vecina, o con sus familiares, en el peor de los casos, dejan bajo la responsabilidad de los hijos mayores, encerrados en sus casas”. Respecto a los factores culturales, los entrevistados manifestaron conocer la cultura de higiene para preparar los alimentos; sin embargo, el problema surge cuando no hay agua: "el agua no llega al cerro". Por tanto, se ve la disyuntiva de comprar agua a la cisterna, pero a veces no va por varios días. Por otro lado, los entrevistados están de acuerdo con que los alimentos nutritivos y ricos en hierro son hígado, sangrecita, bazo, carne roja, pescado azul; sin embargo, se encontró también opiniones como "a mi hijo le doy de comer todo lo que sea rojo, porque ahí está el hierro”; aunque, el factor del color de los alimentos no siempre contiene hierro; por ejemplo, la fresa (Fragaria sp.) es roja, pero no tiene hierro; la tuna (Opuntia ficus-indica); la betarraga (Beta vulgaris); el pimentón (Capsicum annuum). Además, identificaron a los alimentos complementarios al hierro y su necesidad en la dieta de los niños: verduras, menestras, frutas, cítricos, pescado, pollo, lentejas, leche, huevos, queso, quinua. Por último, están los horarios de comidas "el horario de comida para los niños / as es diferente a los de un adulto".

En lo referente a la toma de decisiones sobre la desnutrición crónica, los entrevistados manifestaron que su participación, mediante los diálogos ciudadanos en salud, es un medio de comunicación con el Estado (una herramienta de gestión), que no solo permite hacer un diagnóstico social, sino abordar de manera inmediata las necesidades de la población, de modo que ayuda en su desarrollo humano. Por otro lado, se logra percibir también los diferentes factores determinantes en la alimentación de los niños menores de cinco años, con desnutrición crónica: mitos o creencias culturales, situaciones de pobreza, cuidados por parte de terceros a los niños, entre lo más relevantes. La participación ciudadana permite la focalización en un tema específico, la desnutrición crónica; además de reflejar su pertinencia, es una herramienta de gestión pública que toma en consideración la realidad social. Por otra parte, hubo muchos entrevistados que mostraron escepticismo a la efectividad del diálogo ciudadano como herramienta que podría mejorar la gestión en la salud pública, señalando que no es la primera vez que se tratan estos temas de manera abierta a la población, sino que en muchos de los establecimientos de salud se han propuesto alternativas de desarrollo nutricional que distan de los convencionales propuestos desde las direcciones del Minsa, pero que cuando llega el momento de ejecutar los programas nutricionales se observa que se proveen las mismas recetas que siempre se han dado. Esto causa rechazo en la población de este distrito, por lo 
Participación ciudadana como estrategia de diagnóstico para la toma de decisiones para reducir la desnutrición crónica en un distrito de Lima

que, la renuencia a las directrices nutricionales no solamente se liga a un tema de cultura sino también a un fraccionamiento de la credibilidad respecto a las sugerencias o propuestas por los pobladores. A la sazón, se debe señalar que dichas propuestas se ligan hacia el empleo de derivados vegetales medicinales como parte de la dieta y que, de manera ancestral, muchos de ellos conocen desde sus lugares de origen.

\section{Discusión}

La desnutrición crónica comprende la descompensación alimenticia en el organismo humano, malas prácticas de alimentación que a largo plazo repercuten en la acción propia del desarrollo humano en sus primeras etapas de vida. La alimentación es la base para la formación infantil, ella representa prácticas sociales, económicas y culturales comprendiendo una gama de conocimientos estructurados en el arte culinario (Douglas, 2003); pero al mismo tiempo es una de las principales preocupaciones, que aún sigue repercutiendo en la dieta de las familias, específicamente en los niños menores de cinco años. La desnutrición crónica es un flagelo social que no solo repercute en la economía doméstica, sino en el desarrollo de un país. Estas condiciones de vida definidas como determinantes sociales de la alimentación (Wilkinson \& Marmot, 2003; Villar, 2011), requieren de una respuesta por parte de los gestores que tienen la facultad de tomar decisiones frente a un problema social. Considerar la mejor opción es una prioridad ante una epidemia como la desnutrición crónica.

Los resultados dan cuenta de las enormes brechas que se originan en la gestión de los servicios de salud, una constante frente a un flagelo social, manifestada por la insatisfacción de los usuarios. En este sentido, lograr la eficiencia y eficacia permite generar excedentes en el crecimiento institucional y por ende empodera a las personas que la componen (Madero, 2012). Conocer sobre la pertinencia de la participación ciudadana como estrategia en la toma de decisiones para reducir la desnutrición, es importante, porque el diálogo ciudadano es un medio de comunicación entre la ciudadanía y el Estado, un canal para expresar las percepciones referentes al servicio que prestan las instituciones públicas, una herramienta de gestión que ha permitido hacer un diagnóstico social, además de haberse sentido considerados, los ciudadanos son protagonistas del desarrollo territorial (Peyré, 2016). Afirmar la participación de la ciudadanía es reconocer los derechos ciudadanos y la responsabilidad para con el Estado, este último debe involucrar a la población (Hintze, 2017) en las políticas públicas de salud, sean estos representados en la sociedad civil, como profesionales sanitarios o autoridades de todo nivel de gobierno.

La participación ciudadana es un termómetro de fortalecimientos entre el Estado y la ciudadanía, bajo niveles de gestión, normativas reguladoras en su implementación, arreglos institucionales con la participación ciudadana en materia de los servicios de salud y los directivos, gestores de la administración pública (Delamaza, 2011). Empero, es fundamental que la gerencia planifique, 
mantenga coherencia consigo misma y distribuya los recursos del Estado entre los ciudadanos (Solano, 2003). No obstante, el riesgo de dar un doble discurso conllevaría a un deterioro aún mayor de la desconfianza y escepticismo manifestada por los entrevistados, puesto que existe probabilidad de generar los recursos para la atención, pero al mismo tiempo podría dilatarse la ejecución de los planes en el tiempo, postergando las oportunidades para reducir las tasas de desnutrición. Esta situación, además de la manifestada desconfianza y descrédito institucional, podría generar resentimiento social que podría traducirse en movilizaciones y desorden con una muralla muy fuerte de no credibilidad ciudadana. Ante esto, se hace imprescindible que la participación ciudadana sea conducida responsablemente, sin caer en eventuales oportunidades de populismo ni sea usado por gobernantes de turno para el logro de incremento de simpatizantes con claras intenciones de proselitismo político.

Por otro lado, los determinantes sociales de la alimentación denotaron tres factores determinantes: aspectos sociales, culturales y económicos, los mismos que influyen en las dinámicas sociales de la población vulnerable de San Juan de Lurigancho, que padece la desnutrición crónica. Frente a ello, es necesario dotar de información a la población para el uso de los micronutrientes, supervisar la eficiencia del programa de crecimiento y desarrollo (CRED) en concordancia con las campañas educativas y promoción de la salud, acciones que se deben fomentar dentro de los colegios, en los espacios de la escuela de padres, sin dejar de lado, la participación de personas con discapacidad (Vértiz, Pérez, Faustino, Vértiz y Alain, 2019). Asimismo, el énfasis del análisis del aspecto cultural requiere de la incursión de otros profesionales a los equipos de salud, debido a que la comprensión sociocultural requiere de personas instruidas para ello. Esta recomposición de los equipos de salud proveerá de mayores y mejores herramientas para llevar a cabo de manera efectiva esta estrategia de gestión en el sector salud.

\section{Conclusión}

La participación ciudadana es potencialmente una excelente herramienta para emplearse como estrategia que proporcione mejores elementos de juicio a los gestores en salud, sin embargo para el logro de su efectividad debe considerarse la multidisciplinariedad en los equipos salubristas; retomar la credibilidad de los ciudadanos como un punto de inicio; crear canales sinceros de comunicación entre los ciudadanos y los directivos públicos del sector; priorizar la institucionalidad del cumplimiento de las políticas públicas sobre toda circunstancia o coyuntura proselitista de turno; hurgar en nuevos modos de nutrición basada en la investigación y sistematización de los saberes ancestrales provenientes de la información cultural de la población, pudiendo emplear al diálogo ciudadano como instrumento para identificar a los factores determinantes de la alimentación ciudadana y finalmente, articulando los esfuerzos del sector salud con los demás sectores estatales (educación, cultura, desarrollo e inclusión social, economía, etc.). 
Participación ciudadana como estrategia de diagnóstico para la toma de decisiones para reducir la desnutrición crónica en un distrito de Lima

\section{Agradecimientos}

A los funcionarios de la DIRIS Lima Este, a los 25 agentes comunitarios de salud y a los 18 voluntarios que participaron en la investigación.

\section{Referencias}

Aparco, J. P., Bautista, W., Astete, L. y Pillaca, J. (2016). Evaluación del estado nutricional, patrones de consumo alimentario y de actividad física en escolares del Cercado de Lima. RevPeruMedExp Salud Pública, 33(4), 633-639. doi: 10.17843/rpmesp.2016.334.2545.

Barbour, R. (2013). Los grupos de discusión en investigación cualitativa. Madrid, España: Ediciones Morata.

Bonome, M. (2009). La racionalidad en la toma de decisiones análisis de la teoría de las decisiones, análisis de la teoría de la decisión de Herbert Simon. Recuperado de https://core.ac.uk/ download/pdf/61909687.pdf.

Broeder, L., Uiters, E., Have,W.,Wagemakers,A. \& Schuit,A. (2017). Community participation in Health Impact Assessment. A scoping review of the literatura. Environmental Impact Assessment Review, 66, 33-42. doi: http: / /dx.doi.org/10.1016/j.eiar.2017.06.004.

Castro, M. (2014). Toma de decisiones asertivas para una gerencia efectiva. Recuperado de https: / / repository.unimilitar.edu.co/handle/10654/11746.

Cediel, G., Olivares, M., Brito,A., Cori, H. \& López, D. (2015). Zinc Deficiency in LatinAmerica and the Caribbean. Food Nutr Bull., 36(2), S129-38. doi: 10.1177/0379572115585781.

Congreso de la República. (2002). Acervo documentario en línea. Ley Orgánica de Gobiernos Regionales, Perú. Recuperado de https://bit.ly/2du3Y1E.

Congreso de la República. (2003). Acervo documentario en línea. Ley de los Derechos de Participación y Control Ciudadanos, Perú. Recuperado de https: / / bit.ly/29UbHaD.

De la Cruz, J. C., Figueredo, R., Dugloszewski, J., Díaz, R., Spolidoro, J. y Mato, A. (2008). Declaración de Cancún: declaración internacional de Cancún sobre el derecho a la nutrición en los hospitales. Nutr.Hosp., 23(5), 413-417. Recuperado de https://bit. ly/2MwSzQt.

Delamaza G. (2011). Espacio público y participación ciudadana en la gestión pública en Chile: límites y posibilidades. Polis, 30, 1-28. Recuperado de https: / bit.ly/2X3hj5V.

Douglas, M. (2003). Food in the social order. Studies of food and festivities in three American Communities. Routledge, London: Psychology Press. 
Driss, O., Mellouli, S. \& Trabelsi, Z. (2018). From citizens to government policy-makers: social media data analysis. Government Information Quarterly. Recuperado de https://doi. Federación Latinoamericana de Terapia Nutricional, Nutrición Clínica y Metabolismo FELANPE-. (2008). Declaración internacional sobre el derecho a la nutrición en los hospitales. Cancún, México: Comité Ejecutivo FELANPE.

Flick, U. (2004). Diseño de la investigación cualitativa. Madrid, España: Ediciones Morata.

Font, J., Blanco, I., Gomá, R. y Jarque, M. (2000). Mecanismos de participación ciudadana en la toma de decisiones locales: una visión panorámica. En M. Canto. (Ed.), Participación ciudadana en las políticas públicas. Ciudad de México, México: Siglo XXI editores.

Franco, M., Diez, J., Gullón, P., Margolles, M., Cofiño, R., Pasarín, M. \& Borrell, C. (2018). Towards a policy relevant neighborhoods and health agenda: engaging citizens, researchers, policy makers and public health professionals. GacSanit., 32(S1), 69-73. Recuperado de https://doi.org/10.1016/j.gaceta.2018.07.002.

Gambara, H. y González, E. (2004). Qué y cómo deciden los adolescentes. Tarbiya, 34. En E. Vargas., J. Henao. y C. González. (2007). Toma de decisiones sexuales y reproductivas en la adolescencia. Acta Colombiana de Psicología, 10(1), Recuperado de https: / /www.redalyc. org/articulo.oa?id=798/79810106.

García, R. y Gálvez, N. (2016). Calidad de atención asociada a la satisfacción del usuario externo en los establecimientos asistenciales de salud: Minsa-Essalud, Chongoyape. Rev. Tzhoeco, 8(2). Recuperado de http://revistas.uss.edu.pe/index.php/tzh/article/view/383.

Gómez, M. E., Pastor, G., Pérez, O. e Iriarte, M. T. (2017). Análisis de las buenas prácticas en las unidades de gestión del Servicio Andaluz de Salud. Gac Sanit., 31(2), 139-144. doi: http: / /dx.doi.org/10.1016/j.gaceta.2016.06.003.

Halperin, L. (2003). El contrato social o principios de derecho político. Jean - Jacques Rousseau. Recuperado de https: / / bit.ly/2wevWHm.

Heidegger, M. (2008). Introducción a la investigación fenomenológica. Madrid, España: Síntesis.

Hernández, A., Ruano, A., Hurting, A., Goicolea, I., San Sebastián, M. \& Flores, W. (2019). Pathways to accountability in rural Guatemala: A qualitative comparative analysis of citizen-led initiatives for the right to health of indigenous populations. World Development, 113, 392-401. doi: https:// doi.org/10.1016/j.worlddev.2018.09.020.

Hintze, C. M. (2017). El rol de los espacios de concertación y la participación ciudadana en el desarrollo local: El caso del distrito de Paucartambo, Cusco, entre 2007-2012 (tesis de grado). Lima, Perú, Pontificia Universidad Católica del Perú.

Hoon-Chuah, F., Srivastava, A., Singh, S., Haldane, V., Choon Huat Koh, G., Seng, C., McCoy, D. \& Legido-Quigley, H. (2018). Community participation in general health initiatives 
in high and upper middle-income countries: A systematic review exploring the nature of participation, use of theories, contextual drivers and power relations in community participation. Social Science \& Medicine, 213, 106-122. Recuperado de https://doi. org/10.1016/j.socscimed.2018.07.019.

Instituto Nacional de Estadística e Información. (2017). Perú: Perfil sociodemográfico Informe nacional, censos nacionales 2017, XII de población,VII de vivienda y III de comunidades indígenas. Recuperado de https://bit.ly/2NZze8D.

Jo, N., Marín, D. y Puicón, C. (2018). Prevalencia del sobrepeso y obesidad en niños y adolescentes a grandes altitudes del Ande peruano. Rev. Fac. Med. Hum., 18(4), 84-91. doi: 10.25176/RFMH.v18.n4.1735.

Koontz, H. y Weihrich, H. (2013). Elementos de administración, un enfoque internacional y de innovación. Ciudad de México, México: McGraw Hill Education.

Lasswell, H. (1974). Some perplexities of policy theory. Social Research, (41), 176-189. Recuperado de https://bit.ly/33SwexX.

López, V., Segura, J., Pires, M. P., Malmusi, D., Vergara, M. y Pérez, E. (2018). Municipalismo y salud comunitaria: transformar desde los ayuntamientos. Rev. GacSanit., 32(S1), 26-31. Doi: 10.1016/j.gaceta.2018.06.007.

Madero, S. (2012). La efectividad de las compensaciones, la satisfacción del trabajador y las dimensiones del ambiente laboral. Ciencia UANL, 15(57), 93-100. Recuperado de https: / / dialnet.unirioja.es/servlet/articulo?codigo $=3835381$.

Martínez, R. y Fernández, A. (2009). El costo del hambre: impacto social y económico de la desnutrición infantil en el Estado Plurinacional de Bolivia, Ecuador, Paraguay y Perú. Santiago de Chile, Chile: Naciones Unidas Cepal.

Matos, J. (2012). Perú Estado desbordado y sociedad nacional emergente. Lima, Perú: Universidad Ricardo Palma.

Mearin, F. (2018). Living without anaemia. Gastroenterol.Hepatol., 41(4), 223-225. Doi: 10.1016/j.gastre.2018.04.014.

Ngo, J. \& Serra-Majem, L. (2017). Hunger and Malnutrition. Module in Food Science. Doi: 10.1016/B978-0-08-100596-5.22064-7.

Ooms, G., Keygnaert, I. \& Hammonds, R. (2019). The right to health: from citizen's right to human right (and back). Public Health, 172, 99-104. Doi https://doi.org/10.1016/j. puhe.2019.01.019.

Padmaja, R., Pramanik, S., Pingali, P., Bantilan, C. \& Kavitha, K. (2019). Understanding nutritional outcomes through gendered analysis of time-use patterns in semi-arid India. Global Food Security, 23(2), 49-63. Doi: https: / / doi.org/10.1016/j.gfs.2019.04.001. 
Peyré, I. (2016). Innovaciones sociales e inclusivas: límites y posibilidades para el desarrollo territorial en el contexto de la globalización. Rethinking Clusters, II InternationalWorkshop on Cluster Research - 15/17 th May 2019 - University of Padova, Italy. Doi: 10.13140/ RG.2.1.4271.8320.

Poloni, J. (1987). San Juan de Lurigancho: su historia y su gente: un distrito popular de Lima. Lima, Perú: Centro de Estudios y Publicaciones.

Ravasco, P., Anderson, H. y Mardones, F. (2010). Métodos de valoración del estado nutricional. Nutr Hosp., 25(supl.3), 57-66. Recuperado de https:/ / bit.ly/2YEG7B8.

Reeves, A. \& Mackenbach, J. (2019). Can inequalities in political participation explain health inequalities? Social Science \& Medicine, 234(9), 112371. Recuperado de https://doi. org/10.1016/j.socscimed.2019.112371.

Robbins, S. \& Judge, T. (2013). Organizational Behavior. Paris, France: Pearson - University of Notre Dame.

Rodríguez, R., Pedraja, L. y Araneda, C. (2013). El proceso de toma de decisiones y la eficacia organizativa en empresas privadas del norte de Chile. Ingeniare, 21(3), 328-336. doi: http://dx.doi.org/10.4067/S0718-33052013000300003.

Sabbahi, M., Li, J., Davis, C. \& Downs, S. (2018). The Role of the Sustainable Development Goals to Reduce the Global Burden of Malnutrition. Advances in Food Security and Sustainability, 3, 211-333. Doi: https://doi.org/10.1016/bs.af2s.2018.09.007.

Sartori, G. (2009). Teoría de la democracia: el debate contemporáneo. Madrid, España: Alianza Universidad.

Seligman, M. (2011). Florecer. La nueva psicología positiva y la búsqueda del bienestar. Ciudad de México, México: Océano Expréss.

Serapioni, M. \& Matos, R. (2014). Citizen participation and discontent in three Southern European health systems. Social Science \& Medicine, 123, 226- 233. doi: 10.1016/j. socscimed.2014.06.006.

Simon, H. (1984). La nueva ciencia de la decisión gerencial. Buenos Aires, Argentina: El Ateneo.

Sistema Peruano de Información Jurídica. (2003). Acervo documentario en línea. Ley Orgánica de Municipalidades. Recuperado de https://bit.ly/2yvEGd7.

Solano, A. I. (2003). Toma de decisiones gerenciales. Revista Tecnología en Marcha, 16(3), 44-51. Recuperado de https://revistas.tec.ac.cr/index.php/tec_marcha/article/view/1467

Stoner, J., Freeman, R. \& Gilbert, D. (2003). Administration. Nuevo México: Pearson Education.

Timasheff, N. (1961). La teoría sociológica, su naturaleza y desarrollo. Ciudad de México, México: Fondo de Cultura Económica. 
Unicef. (2015). The faces of malnutrition. Recuperado de https: / / uni.cf/31vdHe3.

Valles, M. (1990). Entrevistas cualitativas. Madrid, España: Centro de Investigaciones Sociológicas.

Vértiz, R., Pérez, S., Faustino, M., Vértiz, J. y Alain, L. (2019). Tecnología de la Información y Comunicación en estudiantes del nivel primario en el marco de la educación inclusiva en un Centro de Educación Básica Especial. Propósitos y Representaciones, 7(1), 1-11. doi: http: / /dx.doi.org/10.20511/pyr2019.v7n1.266.

Villar, M. (2011). Factores determinantes de la salud: importancia de la prevención. Acta Med. Per., 28(4), 237-241. Recuperado de http://www.scielo.org.pe/pdf/amp/v28n4/a11. pdf.

Ward, J., Cafiero, F., Fretigny, R., Colgrove, J. \& Seror, V. (2019). France's citizen consultation on vaccination and the challenges of participatory democracy in health. Social Science \& Medicine, 220, 73-80. Recuperado de https://doi.org/10.1016/j. socscimed.2018.10.032.

Wilkinson, R. \& Marmot, M. (2003). Social determinants of health, The Solid Facts. Denmark: WHO Library Cataloguing Publication Data.

World Organization Health -WHO-. (2014). Plan de aplicación integral sobre nutrición materna, del lactante y del niño pequeño. Recuperado de https://www.who.int/nutrition/ publications/CIP_document/es/. 